\title{
Multi-scale finite element model validation method of cable-stayed bridge based on the support vector regression
}

\section{Pei Juan Zheng, Zhou Hong Zong*, Qi Qi Liu, Jie Niu, Hai Fei Zhou and Ru Mian Zhong}

Department of Civil Engineering,

Southeast University,

Nanjing, 211189, China

Email: peijuanzheng1987@163.com

Email: zongzh@seu.edu.cn

Email: 550654647@qq.com

Email: 370389258@qq.com

Email:675029028@qq.com

Email: 371806766@qq.com

*Corresponding author

\begin{abstract}
In this paper, the multi-scale finite element model (FEM) of a composite cable-stayed bridge, Guanhe Bridge, was established based on the Arlequin method firstly. Then a two-step multi-scale FE model updating method was proposed. Furthermore, based on structural health monitoring (SHM) system of Guanhe Bridge, support vector regression (SVR) method was employed to analyse the uncertainty quantification and transmission. It was shown that the errors between the calculated frequencies from the updated multi-scale FEM and the measured frequencies from SHM were less than 3\%. In the procedure of inverse uncertainty propagation, the coincidence indexes of the structural parameters were larger than $65 \%$. The deviations between the optimal values of the updated parameters and the corresponding statistical mean values were very small $(<5 \%)$. Finally, the analysis results indicate that the distributions of the parameters agree well with the assumed normal distribution.
\end{abstract}

Keywords: multi-scale simulation; finite element model; FEM; validation; support vector regression; SVR; uncertainty quantification and propagation; composite cable-stayed bridge.

Reference to this paper should be made as follows: Zheng, P.J., Zong, Z.H., Liu, Q.Q., Niu, J., Zhou, H.F. and Zhong, R.M. (2019) 'Multi-scale finite element model validation method of cable-stayed bridge based on the support vector regression', Int. J. Lifecycle Performance Engineering, Vol. 3, No. 1, pp.35-58.

Biographical notes: Pei Juan Zheng is a $\mathrm{PhD}$ student from the Bridge Structural Health Monitoring Analysis.

Zhou Hong Zong is a $\mathrm{PhD}$ /Professor from the Bridge Structural Health Monitoring Analysis and Safety Prognosis.

Qi Qi Liu received his Master's from the Bridge Structural Health Monitoring Analysis. 
Jie Niu is a PhD student from the Bridge Structural Health Monitoring Analysis.

Hai Fei Zhou is a PhD student from the Bridge Structural Health Monitoring Analysis.

Ru Mian Zhong is a PhD student from the Bridge Structural Health Monitoring Analysis.

\section{Introduction}

The structural health monitoring (SHM) systems of bridges provided reliable references for the operation condition assessment and prognosis of the bridges recently (Farrar and Lieven, 2007; Ou and Li, 2010). However, for the large span bridges, the traditional single-scale finite element model (FEM) cannot be adopted to consider the different scale effect in the damage identification process. So it is one of the basic steps for safety prognosis of bridges to build a multi-scale FEM which contained the information of both the whole structures and their local details and validate the precision of this multi-scale model (Zong et al., 2014).

It is an important method to employ multi-scale simulation to solve the complicated materials and engineering problems. Many factors, for instance, the different scales, coupling correlation scales, are taken into consideration in order to improve the simulation efficiency and obtain the more useful microcosmic information. In recent years, this simulation method was applied into the analysis of the whole and local structural properties and its application also got some results. For example, Yalchin et al. introduced the theory and applications of multi-scale finite element (FE) methods (Efendiev and Hou, 2009). Michaels et al. (2012) discussed in detail the application of the damage prognosis based on multi-scale model in SHM system of the aircraft. Starge (2010) proposed that the three critical problems of the multi-scale damage prognosis were multi-scale simulation of the materials, the certainty of multi-scale simulation and the quantisation of model-form uncertainty. Greco et al. (2015) adopted the multi-scale technique to analyse the crack propagation in composites. Yang (2012) presented the multi-scale simulation of the large span bridges. Liu et al. (2010) adopted the virtual internal force method to achieve multi-scale connection in the joint interfaces and implemented the adaptive computation. Perera et al. (2013) proposed the multi-scale structural damage identification procedures by combining the static and dynamic measurements. Ding et al. (2010) used multi-scale numerical computation to analyse the damage of a long-span cable-stayed bridge. Takizawa and Tazduyar (2011) provided the multi-scale space-time techniques for fluid-structure interaction computations.

How to employ the measured response in order to assess the precision of the traditional and multi-scale FEM was the main part of FEM updating and validation (Oberkampf and Roy, 2010; Marwala, 2010; Jaishi and Ren, 2007). Sandia National Laboratories Validation Workshop gave some broad guidelines for the model validations of structural dynamics in 2006 (Red-Horse and Paez, 2008). In the following year, they summarised the structural dynamics challenge problems and proposed that the concerned issues involved the development of a mathematical framework for uncertainty 
quantification of structures and the validation of the corresponding mathematical model (Paez and Red-Horse, 2008). Generally, the uncertainty analysis contains random uncertainty and cognitive uncertainty. The former is related to the randomness of the events. The other one is caused by a lack of corresponding model data and knowledge. Moreover, some special software such as NESSUS and DAKOTA, have been developed for the uncertainty analysis. But they always focus on the random uncertainty and cannot be adopted to reduce the cognitive uncertainty to some extent (Catbas et al., 2013). So it is very important for the continuous model updating to employ the long-term monitoring data (Wei and Dyke, 2014).

Uncertainty quantisation is a critical component of computational dynamics and experimental mechanics (Montomoli et al., 2015; Batou et al., 2015; Atamturktur et al., 2015). Monte Carlo simulation, Statistical hypothesis testing and Bayesian hypothesis testing-based methods are always used to solve this problem (Smith and Doyle, 1992; Smith, 1995; Thompson et al., 1999; Renno and MacE, 2012; Liu et al., 1988; Hemez and Doebling, 2001; Mak et al., 2012; Adhikari and Sarkar, 2009; Chen et al., 2004). For this question, Nishio et al. (2012) discussed the uncertainty quantification rules for the modelling verification and validation $(\mathrm{V} \& \mathrm{~V})$ of the existing and aging bridges by applying the $\mathrm{V} \& \mathrm{~V}$ procedures based on Bayesian inference into one bridge. Ren and Chen (2010) proposed a response surface-based FEM updating procedure for civil engineering structures in structural dynamics and applied the method into the FE model updating of a full-size bridge. Moradi et al. (2010) presented a method which employed bees algorithm (BA) in the FE model updating of structures. In addition, Xiao et al. proposed a multi-scale model updating method based on model frequencies and influence lines and then applied it to the multi-scale FE model updating of the Stonecutters Bridge (Xiao et al., 2015).

It is a critical problem for the multi-scale model development to quantify the uncertainty of multi-scale $\mathrm{V} \& \mathrm{~V}$ problems and it is also considered to be the key factor to solve the technical problems of SHM. However, at present, the researches about model validation are mostly for the traditional FEM and there are less works on the multi-scale model updating and validation. Zhong et al. $(2013,2015)$ presented a multi-scale FEM updating method of large-span cable-stayed bridges based on two-phase response surface method and its application proved that it can be adopted further for the multi-scale damage detection and multi-scale damage prognosis. Zhong et al. (2016) provided a method for multi-scale FEM validation based on the two-step multi-scale FE model updating method and probability box (P-box) theory. Chan et al. (2009) proposed the general procedures of multi-scale model updating and verification for nonlinear physical-based modelling of large civil infrastructure and applied this method to the model verification of a long-span bridge.

In this paper, firstly, the Arlequin method (Ben, 1998) is introduced to explore the mathematical description of structural multi-scale FEM. Then polynomial response surface method and support vector regression (SVR) method are adopted to establish the basic framework of two-phase FEM updating and validation in order to reduce the model errors and parameter errors. Above all, a reverse surrogate model between structural parameters for bridges and the corresponding structural responses is built by the application of SVR method. This indicates that the reverse propagation rules of the uncertainty are carried out. Finally, in order to evaluate the feasibility of the proposed 
method, it is adopted to realise the work of the multi-scale model validation of a cable-stayed bridge, Guanhe Bridge.

\section{Multi-scale simulation of cable-stayed bridge}

\section{$2.1 \quad$ Multi-scale structural simulation method}

In this paper, the Arlequin method (Ben, 1998) (Table 1) is adopted to build the multi-scale model. It contains the following basic theory (Qiao et al., 2011):

1 The whole structure consists of two parts, the uncoupled area $\Omega_{1}$ and the non-overlapping domain $\Omega_{g}$.

2 The weight functions, $\alpha_{i}$ and $\beta_{i}$, are defined to solve the energy distribution problems in the overlapping domain of different models.

3 Overlapping operator is defined in the domain $\Omega_{g}$.

Figure 1 Arlequin method

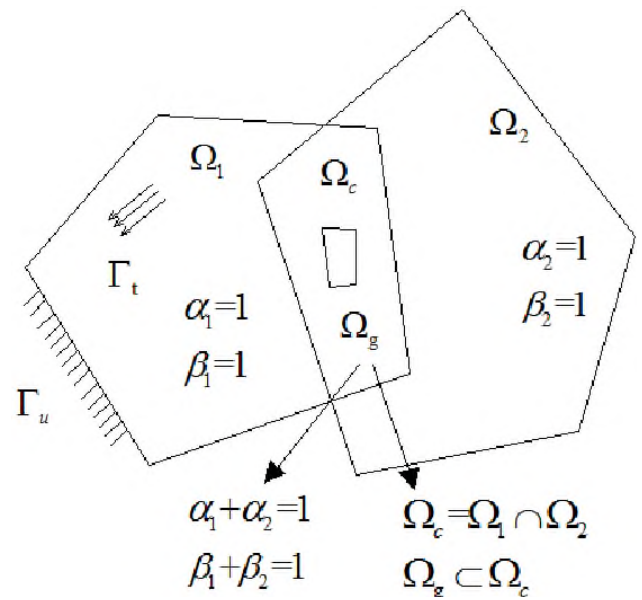

As shown in Figure 1, structural multi-scale simulation can be expressed by adopting the Lagrange multiplier field which is obtained by the discretisation of 'virtual element $\lambda$ ' in the coupling zone $\Omega_{g}$ to couple the coarse domain $\Omega_{1}$ and the fine domain $\Omega_{2}$. The static equilibrium equations can be given by

$$
\left[\begin{array}{ccc}
\alpha_{1} K_{1} & 0 & C_{\lambda 1}^{T} \\
0 & \alpha_{2} K_{2} & -C_{\lambda 2}^{T} \\
C_{\lambda 1} & -C_{\lambda 2} & 0
\end{array}\right]\left\{\begin{array}{l}
x_{1} \\
x_{2} \\
x_{\lambda}
\end{array}\right\}=\left\{\begin{array}{c}
F_{1} \\
F_{2} \\
0
\end{array}\right\}
$$

where $K_{1}$ and $K_{2}$ are the element stiffness matrix. $F_{1}$ and $F_{2}$ form the load matrix. $x_{1}, x_{2}$ and $x_{\lambda}$ represent the coordinate values of basis function for FEM. $C_{\lambda 1}$ and $C_{\lambda 2}$ are the coupling matrix between the virtual element $\lambda$ and the coarse element 1 , the fine element 2 respectively. On the same way, the dynamic equilibrium equations also can be obtained by 


$$
\begin{aligned}
& {\left[\begin{array}{ccc}
\alpha_{1} K_{1} & 0 & C_{\lambda 1}^{T} \\
0 & \alpha_{2} K_{2} & -C_{\lambda 2}^{T} \\
C_{\lambda 1} & -C_{\lambda 2} & 0
\end{array}\right]\left\{\begin{array}{l}
x_{1} \\
x_{2} \\
x_{\lambda}
\end{array}\right\}+\left[\begin{array}{ccc}
\alpha_{1} C_{1} & 0 & 0 \\
0 & \alpha_{2} C_{2} & 0 \\
0 & 0 & 0
\end{array}\right]\left\{\begin{array}{l}
\dot{x}_{1} \\
\dot{x}_{2} \\
\dot{x}_{\lambda}
\end{array}\right\}} \\
& +\left[\begin{array}{ccc}
\alpha_{1} M_{1} & 0 & 0 \\
0 & \alpha_{2} M_{2} & 0 \\
0 & 0 & 0
\end{array}\right]\left\{\begin{array}{l}
\ddot{x}_{2} \\
\ddot{x}_{2} \\
\ddot{x}_{\lambda}
\end{array}\right\}=\left\{\begin{array}{c}
F_{1} \\
F_{2} \\
0
\end{array}\right\}
\end{aligned}
$$

The coupling matrix can be gotten by the Gauss integral. After that, the static and dynamic analysis for structures can be realised. However, it is very tedious to obtain $C_{\lambda i}$ and carry out the secondary development of the FE software in this process. So this problem will restrict the application and generalisation of Arlequin method for structural multi-scale simulation. In this paper, the iterative approximation theory in the FEM updating is used to avoid the mathematical derivation of the overlapping matrix and obtain the unknown parameters of the above equations in the application of the Arlequin method. These parameters contain the weight factors $\alpha_{1}, \alpha_{2}$ in coupling domain and the overlapping matrix $C_{\lambda i}$.

For the structures, the internal force of a beam in a specific area is generally supposed to be carried out by using the interlocking force between the materials of the different elements. For example, it is common for steel-concrete composite bridges to adopt the shear connector and longitudinal connection of the post-poured zones to make the different elements work together. The coupling interaction of the beam/shell elements is shown in Figure 2. Similarly, in the structural multi-scale simulation, it is assumed that the different elements with the same materials in the overlapping area connect and co-operate with each other. They use the certain distribution coefficients, $\alpha_{1}$ and $\alpha_{2}$ $\left(\alpha_{1}+\alpha_{2}=1\right)$, to make the structural stiffness and mass, $K$ and $M$, divide into two pieces in this process. In the same way, the connection stiffness in the coupling area is provided with the interlocking force between the materials.

Figure 2 Coupling interaction of beam/shell elements

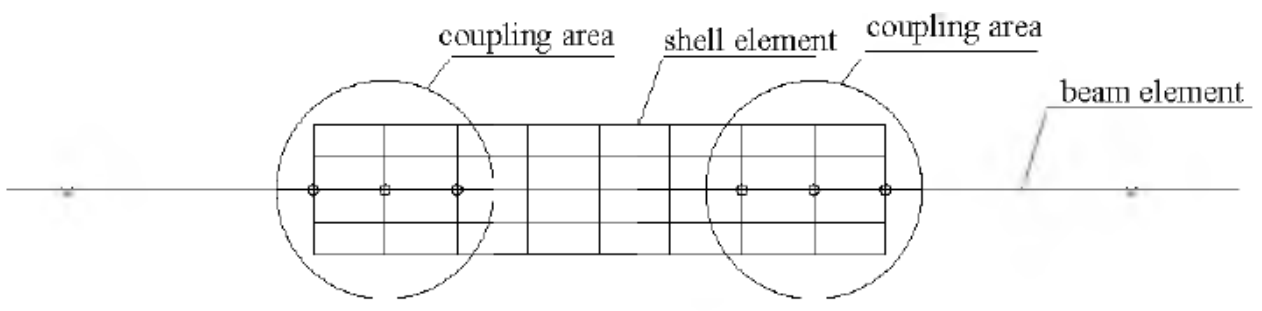

\subsection{Multi-scale FEM simulation of Guanhe Bridge}

Guanhe Bridge, a composite cable-stayed bridge with the spans $32.9 \mathrm{~m}+115.4 \mathrm{~m}+$ $340 \mathrm{~m}+115.4 \mathrm{~m}+32.9 \mathrm{~m}$, is given in Figure 3. It is located in the National G15 expressway in China.

Its traditional FEM and multi-scale FEM were built by employing the FE software, Ansys. In the multi-scale model, three cross beams $(3.9+3.9+3.9=11.7 \mathrm{~m})$ in the mid-span were chosen to be the small-scale area. For the two FEMs, the cables were 
modelled by adopting linear elastic link elements Link8, and the secondary dead load and the saddle weight were simulated using the element Mass21. The restriction effect of the rubber supports was approximated as linear elastic spring elements, Combin14. For the single-scale FEM, the bridge deck is simulated by adopting the shell element, Shell63. The main girder, small girder and the bridge towers were modelled employing the element, Beam188. The simulation of multi-scale model was mostly the same as the single-scale FEM. However, there were some following differences. Firstly, for the multi-scale FEM, the small-scale girder, small longitudinal beam and cross beam is simulated by using the element Shell63. What's more, the bridge deck was modelled adopting the 3D solid element, Solid45. In addition, the non-coupling area of the different elements was simulated by using the spring element (Combin14). These two FEMs are shown in Figure 4.

Figure 3 Main part of Guanhe Bridge (see online version for colours)

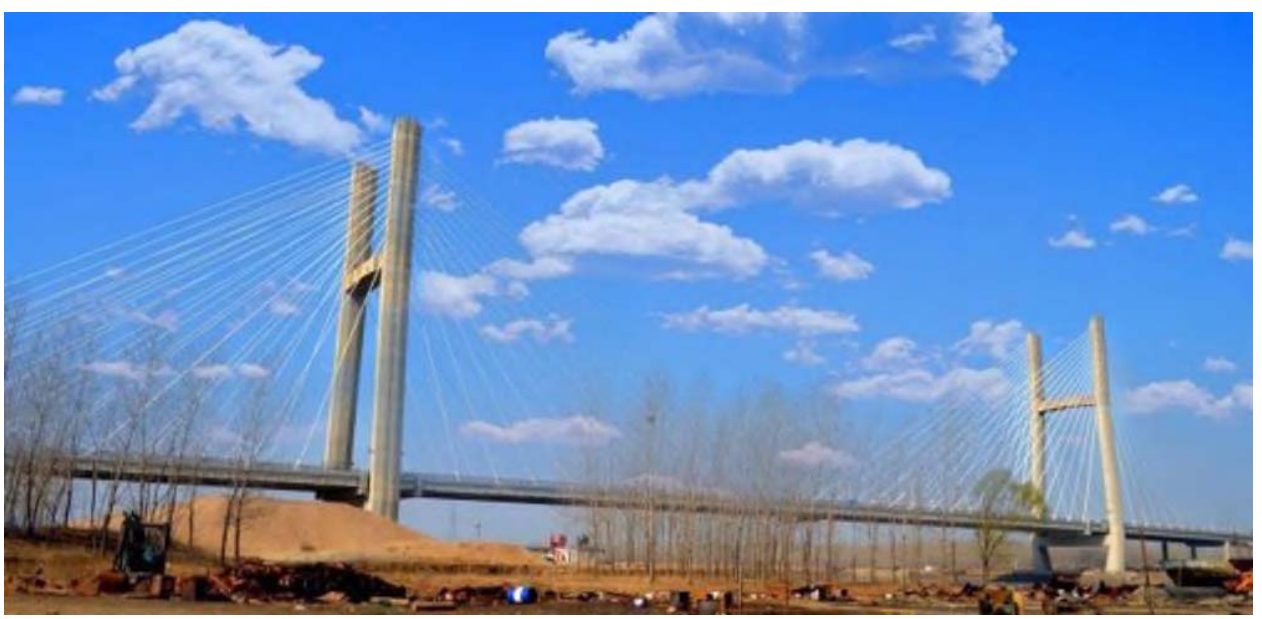

Figure 4 FEMs of Guanhe Bridge, (a) single-scale FEM (b) multi-scale FEM (see online version for colours)

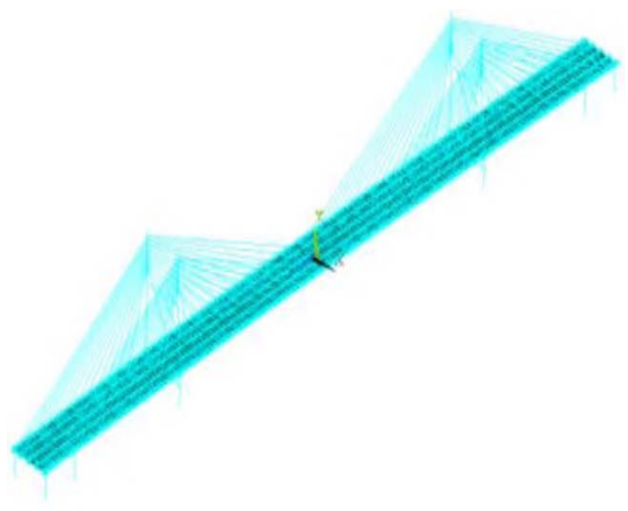

(a)

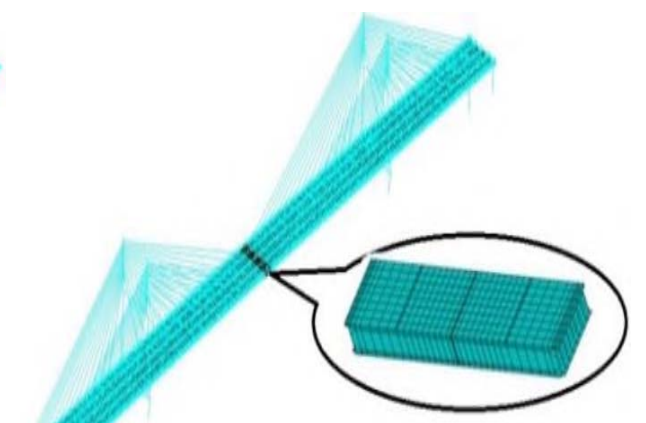

(b) 


\section{Multi-scale FEM updating of cable-stayed bridge}

The multi-scale FEM updating can be divided into two steps. In order to reduce the error of multi-scale FEM simulation, first, the polynomial response surface method (Zong and Ren, 2012) is adopted to update the FEM according to the simulated response of single-scale FEM. Then the following multi-scale FEM updating is based on the SVR method and realised by taking the measured structural response under ambient vibration test as the FEM updating target. In the latter process, the reverse surrogate model is established to decrease the model parameter errors.

\subsection{Multi-scale model-form error updating}

The model error which results from multi-scale coupling effect is taken as the eliminated goal in this updating procedure. So the weight factor a and the vertical, transverse and longitudinal connection stiffness in the coupling area, $K K_{1}, K K_{2}$ and $K K_{3}$, are the unknown parameters. Three-order polynomial response surface method is adopted to carry out the updating process.

\subsubsection{Parameters selection and experiment design}

The small-scale FEM simulation is just realised in the certain area of the mid-span. So in order to decrease the difference between the multi-scale FEM and the target FEM, it is the main problem in this phase to reduce the error which caused by the multi-scale coupling.

Table 1 The range of the updating parameters in multi-scale FEM updating

\begin{tabular}{lcccc}
\hline Parameters & $K K_{1}(\mathrm{~N} / \mathrm{m})$ & $K K_{2}(\mathrm{~N} / \mathrm{m})$ & $K K_{3}(\mathrm{~N} / \mathrm{m})$ & $\alpha$ \\
\hline Maximum & $1 \times 10^{7}$ & $1 \times 10^{7}$ & $1 \times 10^{7}$ & 0.8 \\
Minimum & $1 \times 10^{5}$ & $1 \times 10^{5}$ & $1 \times 10^{5}$ & 0.2 \\
\hline
\end{tabular}

Table 2 Experimental samples and the corresponding simulated structural response based on multi-scale FEM

\begin{tabular}{|c|c|c|c|c|c|c|c|c|}
\hline \multirow{2}{*}{$N$} & \multicolumn{3}{|c|}{ Updating parameters } & \multicolumn{5}{|c|}{ Simulated structural response } \\
\hline & $K K_{1}\left(10^{5}\right)$ & $K K_{2}\left(10^{5}\right)$ & $K K_{3}\left(10^{5}\right)$ & $\alpha$ & $V 2$ & N1 & $N 2$ & $D 1$ \\
\hline 1 & 100.00 & 100.00 & 49.02 & 0.80 & 0.493 & 0.665 & 0.777 & 0.128 \\
\hline 2 & 1.00 & 62.88 & 30.70 & 0.20 & 0.492 & 0.589 & 0.774 & 0.136 \\
\hline 3 & 1.00 & 72.28 & 1.00 & 0.43 & 0.482 & 0.591 & 0.761 & 0.132 \\
\hline 44 & 70.80 & 33.67 & 1.00 & 0.20 & 0.488 & 0.646 & 0.769 & 0.136 \\
\hline 45 & 20.80 & 100.00 & 100.00 & 0.80 & 0.498 & 0.613 & 0.783 & 0.130 \\
\hline
\end{tabular}

Notes: V2 is the second-order vertical mode frequency; N1 and N2 are the first two torsional mode frequencies; D1 is the maximum displacement in the mid-span.

For Guanhe Bridge, the preliminary test results indicate that if the parameters are inappropriate, some mode shapes of the bridge, the second-order vertical mode shape and the first two torsional mode shapes will be un-normal and it will have a great influence on the maximum displacement in the mid-span. So it is necessary to select these simulated 
structural response based on multi-scale FEM as the objective response in order to improve the updating precision of multi-scale FEM. According to the parameter sensitivity analysis and the results of tests (Liu, 2015), the preliminary range of the above parameters is determined and given in Table 1.

After the determination of the range of the above parameters, the experimental design is conducted as follows. The 45 experimental samples are generated by D-optimal design method (Red-Horse and Paez, 2008). Their corresponding structural responses can be obtained by multi-scale FE simulation. The experimental samples and the responses are shown in Table 2.

\subsubsection{Polynomial response surface function fitting}

Normally, second-order polynomial is used to update the FEM of structures (Zong and Ren, 2012). However, the parameters of the second order response polynomial are not sufficient to cover the uncertain parameters of complex structures or the parameters to be modified. In order to improve the precision of response surface model, three-order polynomial is adopted to improve the computational efficiency.

Figure 5 Regression response surface models of target function, (a) second-order vertical response surface model (b) first-order torsional response surface model (see online version for colours)

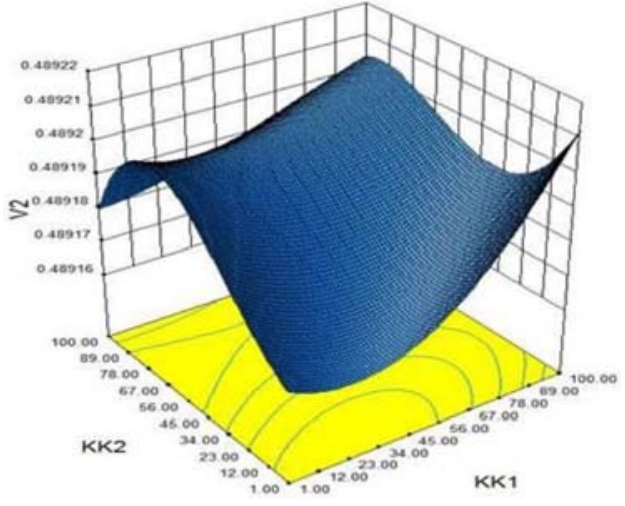

(a)

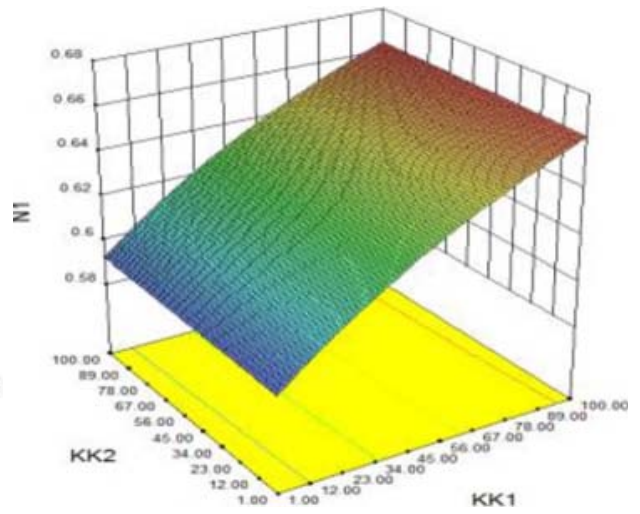

(b)

The response functions of the simulated structural response can be gotten by fitting the experimental samples based on the least square regression analysis method. The second-order vertical and first-order torsional response surface models are shown in Figure 5.

$$
\begin{aligned}
V 2= & 1.36 E-004 \times K K_{1}-2.30 E-009 \times K K_{2} \times K K_{3}+\ldots+1.16 E-009 \times K K_{3}^{3} \\
& +1.29 E-003 \times \alpha^{3} \\
N 1= & +0.58+1.14 E-003 \times K K_{1}+4.57 E-005 \times K K_{2}+0.02 \times \alpha+\ldots+0.01 \times \alpha^{3}
\end{aligned}
$$




\subsubsection{Polynomial response surface model verification}

It is necessary to test the precision of the obtained response surface models and ensure their feasibility. So the indicators $\mathrm{R}^{2}$ and RMSE are adopted (Jaishi and Ren, 2007):

$$
\begin{gathered}
R^{2}=1-\frac{\sum_{j=1}^{N}\left[y_{R S}(j)-y(j)\right]^{2}}{\sum_{j=1}^{N}[y(j)-\bar{y}]^{2}} \\
R M S E=\frac{1}{N \cdot \bar{y}} \cdot \sqrt{\sum\left(y(j)-y_{R S}(j)\right)^{2}}
\end{gathered}
$$

where $y_{R S}, y(j), y(j)$ and $\bar{y}$ are the calculated value of the First-order response surface model, the value of the corresponding FEM and the mean of the FEM respectively. $N$ is the number of the samples in the design space. The calculation results according to the above equations are given in Table $3 . \mathrm{R}^{2}$ and $R M S E$ are very close to 1 and 0 respectively and this shows that there is a little different between the obtained response surface values and the corresponding true parameters. So the response surface model can be employed to reflect the relations between the structural parameters and the corresponding structural response. In other words, the regression response surface model can be used to replace the FEM to accomplish the model updating work.

Table 3 The precision of response surface model

\begin{tabular}{lcl}
\hline Objective function & $R^{2}$ & $R M S E$ \\
\hline 2nd vertical function & 0.9999 & 0.0001 \\
1st torsional function & 0.9998 & 0.0003 \\
2nd torsional function & 0.9998 & 0.0013 \\
Maximum displacement in the mid-span & 0.9923 & 0.0042 \\
\hline
\end{tabular}

In this paper, the single-scale FEM is chosen to be the target FEM. It is noted that the single-scale FEM adopts the same model parameters with those other parameters of multi-scale FEM. Then the chosen corresponding simulated structural response based on the single-scale FEM in Table 4 is obtained and chosen to be the target values of multi-scale FEM optimisation process.

Table 4 Simulated structural response based on single-scale FEM

\begin{tabular}{lcccc}
\hline Model order & $V 2$ & $N 1$ & $N 2$ & $D 1$ \\
\hline Structural response & 0.4885 & 0.5998 & 0.7695 & 0.1320 \\
\hline
\end{tabular}

The multi-object optimisation method is adopted to optimise the established response surface model and complete the first step of multi-scale FEM updating. The iterative optimised parameters are provided in Table 5. The contrast between the simulated structural response after the updated multi-scale FEM and the simulated valves from the sing-scale FEM is given in Table 6. It is shown that the relative errors between the structural responses and the objective ones are no more than $1.1 \%$. In one word, in the 
parameter design space, the error which resulted from multi-scale coupling is reduced to some extent after the multi-scale FEM updating.

Table 5 The initial and updated parameters

\begin{tabular}{lcccc}
\hline Parameters & $K K_{1}$ & $K K_{2}$ & $K K_{3}$ & $\alpha$ \\
\hline Maximum & $1 \times 10^{7}$ & $1 \times 10^{7}$ & $1 \times 10^{7}$ & 0.2 \\
Minimum & $1 \times 10^{5}$ & $1 \times 10^{5}$ & $1 \times 10^{5}$ & 0.8 \\
Mean value & $1 \times 10^{6}$ & $1 \times 10^{6}$ & $1 \times 10^{6}$ & 0.5 \\
Updated value & $1.501 \times 10^{6}$ & $1.375 \times 10^{6}$ & $1.082 \times 10^{6}$ & 0.4 \\
Correction factor (\%) & 50.1 & 37.5 & 8.2 & 20 \\
\hline
\end{tabular}

Table 6 Comparison of the simulated structural responses after updating and the objective ones

\begin{tabular}{lccc}
\hline Parameters $(\mathrm{Hz})$ & $\begin{array}{c}\text { Single-scale simulated } \\
\text { response }\end{array}$ & $\begin{array}{c}\text { Simulated response after } \\
\text { updating }\end{array}$ & Relative error (\%) \\
\hline V2 & 0.4885 & 0.4892 & 0.14 \\
N1 & 0.5998 & 0.6070 & 0.72 \\
N2 & 0.7695 & 0.7705 & 0.13 \\
D1 & 0.1320 & 0.1305 & 1.10 \\
\hline
\end{tabular}

\subsection{Model parameters updating}

Some artificial intelligent methods, for example, neural network method and support vector machine (SVM) method, are developed recently. Though the essence of these methods is response surface method, they can be employed to convert model updating, a traditional inverse problem, to a direct question. The function between the structural responses (independent variables) and the structural parameters (dependent variables) can be expressed by

$$
p=g(y)
$$

Then the optimal parameter of FEM, $\bar{p}$ can be obtained by substituting the measured response $\bar{y}$ into the fitting function.

In this paper, in order to discuss the inverse propagation rules of the parameter uncertainty, SVR method is adopted to take the FEM updating problem as a direct problem and carry out multi-scale FEM updating in the second phase for Guanhe Bridge.

\subsubsection{SVR method}

SVM method was first proposed by Vapnik (1998). It is a machine learning technique based on the statistical learning theory. Its generalisation ability of the learning machine is improved by minimising the structural risk so as to realise the minimisation of the empirical risk and confidence interval. So the less statistical samples can be employed to obtain the good statistical rules in this condition.

Support vector classification (SVC) model and SVR model can be established by adopting the basic theory of SVM method. In this paper, SVR model is introduced simply as follows: 
It is supposed that the samples, $\left(x_{1}, y_{2}\right),\left(x_{2}, y_{2}\right), \ldots,\left(x_{n}, y_{n}\right),\left(x_{1}, y_{1}\right),\left(x_{2}, y_{2}\right), \ldots,\left(x_{n}, y_{n}\right)$, ( $x \in R^{n}$ ), are the sample input. $y \in R$ is used to be the sample output. The aim of support vector machine is to find the suitable function $f(x)=w \cdot \varphi\left(x_{i}\right)+b$ to fit the samples. The error between the prediction values of the function, $f\left(x_{i}\right)$, and the true ones $y_{i}$ can be given using the e-insensitive loss function:

$$
\left|y_{i}-f\left(x_{i}\right)\right|_{\varepsilon}=\max \left\{0,\left|y_{i}-f\left(x_{i}\right)\right|-\varepsilon\right\}
$$

It is considered to be no loss when the samples lie in the band between the two dotted lines which are shown in the Figure 6(a). The band is called e-band. The loss $\xi$ which corresponds to $(\bar{x}, \bar{y})$ in Figure $6(\mathrm{~b})$ can be obtained by $\xi=\bar{y}-f(\bar{x})-\varepsilon$. The regression model of traditional SVM can be given by

$$
\left\{\begin{array}{l}
\min _{w, b} \frac{1}{2}\|w\|^{2} \\
\text { s.t. } \quad y_{i}-\left(w \cdot \varphi\left(x_{i}\right)+b\right) \leq \varepsilon \\
w \cdot \varphi\left(x_{i}\right)+b-y_{i} \leq \varepsilon \quad(i=1,2, \ldots, n)
\end{array}\right.
$$

Figure $6 \varepsilon$-insensitive loss function, (a) $\varepsilon$-band (b) the loss corresponding to $(\bar{x}, \bar{y})$ (see online version for colours)

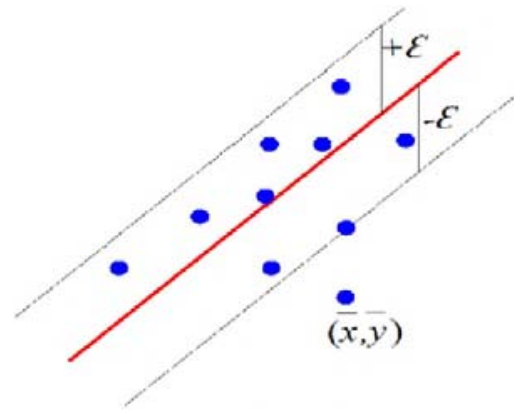

(a)

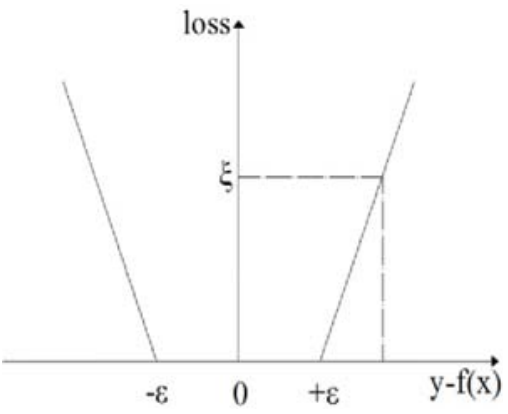

(b)

The more details of SVM method can be found in reference (Vapnik, 1998).

\subsubsection{Parameter selection and experiment design}

The preliminary updating ranges of the parameters are determined according to the sensitivity analysis and the test results (Liu, 2015) and shown in Table 7. However, the order of magnitude for the spring stiffness in the support place can be only confirmed in this process and their updating range is still large.

Since SVM method relies on the samples to learn the whole composite pattern of the problem, it is necessary for the samples to be representative and reflect the problem mode of the different locations in the design space (Montomoli et al., 2015). There are 175 test samples to be generated by adopting the D-optimal experimental design method according to the range of parameters. Their corresponding structural responses can be obtained using FE simulation and they are given in Table 8. Then 150 samples in the whole sample set are chosen to be the learning ones and the other samples are considered 
as the prediction ones. In order to reduce the complexities of the regression function, just the first two vertical and transverse frequencies ( $\mathrm{S} 1, \mathrm{~S} 2$ and $\mathrm{H} 1, \mathrm{H} 2$ respectively) and the first-order longitudinal frequency (Z1) are selected to be the input parameters in the second stage of multi-scale FEM updating based on SVR method. There are eight output parameters $\rho_{1}, E_{1}, E_{2}, \rho_{4}, E_{4}, K_{1}, K_{2}$ and $K_{3}$. In this process, all the input parameters are fitted to obtain each output variable. In other words, the eight SVR functions can be obtained which are the multi-input single-output ones.

Table 7 Parameter selection and updating

\begin{tabular}{llcc}
\hline Updating parameters & & Updating range & Initial parameters \\
\hline Concrete density of bridge deck & $\rho_{1}\left(\times 10^{3} \mathrm{Kg} / \mathrm{m}^{3}\right)$ & $2.25 \sim 2.75$ & 2.5 \\
$\begin{array}{l}\text { Concrete elasticity modulus of } \\
\text { bridge deck }\end{array}$ & $E_{1}\left(\times 10^{10} \mathrm{~N} / \mathrm{m}^{2}\right)$ & $3.45 \sim 3.795$ & 3.45 \\
$\begin{array}{l}\text { Elasticity modulus of steel girder } \\
\text { Concrete density of bridge tower }\end{array}$ & $E_{2}\left(\times 10^{11} \mathrm{~N} / \mathrm{m}^{2}\right)$ & $1.995 \sim 2.31$ & 2.1 \\
$\begin{array}{l}\text { Concrete elasticity modulus of } \\
\text { bridge tower }\end{array}$ & $E^{4}\left(\times 10^{3} \mathrm{Kg} / \mathrm{m}^{3}\right)$ & $2.25 \sim 2.75$ & 2.5 \\
$\begin{array}{l}\text { Transverse spring stiffness in the } \\
\text { side support place }\end{array}$ & $K_{1}\left(\times 10^{6} \mathrm{~N} / \mathrm{m}\right)$ & $3.45 \sim 3.795$ & 3.45 \\
$\begin{array}{l}\text { Transverse spring stiffness in bridge } \\
\text { tower place }\end{array}$ & $K_{2}\left(\times 10^{9} \mathrm{~N} / \mathrm{m}\right)$ & $1 \sim 3$ & 1 \\
Longitudinal spring stiffness & $K_{3}\left(\times 10^{8} \mathrm{~N} / \mathrm{m}\right)$ & $1 \sim 3$ & 1 \\
\hline
\end{tabular}

Table 8 D-optimal experimental design samples and simulated structural responses based on multi-scale FEM

\begin{tabular}{|c|c|c|c|c|c|c|c|c|c|c|c|c|c|}
\hline \multirow{2}{*}{ Number } & \multicolumn{8}{|c|}{ Independent parameters } & \multicolumn{5}{|c|}{ Simulated structural response $(\mathrm{Hz})$} \\
\hline & $\rho_{1}$ & $E_{1}$ & $E_{2}$ & $\rho_{4}$ & $E_{4}$ & $K_{1}$ & $K_{2}$ & $K_{3}$ & S1 & $S 2$ & $H 1$ & $H 2$ & $Z 1$ \\
\hline 1 & 2.25 & 3.45 & 2.15 & 2.75 & 3.69 & 1 & 1 & 1 & 0.384 & 0.503 & 0.341 & 0.461 & 0.786 \\
\hline 2 & 2.63 & 3.79 & 2 & 2.25 & 3.66 & 1 & 1 & 1 & 0.373 & 0.488 & 0.329 & 0.455 & 0.775 \\
\hline 3 & 2.75 & 3.45 & 2.05 & 2.38 & 3.79 & 1 & 1 & 2 & 0.369 & 0.483 & 0.338 & 0.452 & 0.769 \\
\hline$\vdots$ & $\vdots$ & $\vdots$ & $\vdots$ & & $\vdots$ & $\vdots$ & $\vdots$ & $\vdots$ & $\vdots$ & $\vdots$ & $\vdots$ & $\vdots$ & \\
\hline 173 & 2.25 & 3.45 & 2.11 & 2.75 & 3.79 & 3 & 1 & 3 & 0.385 & 0.503 & 0.415 & 0.554 & 0.789 \\
\hline 174 & 2.25 & 3.79 & 2 & 2.75 & 3.79 & 1 & 3 & 1 & 0.384 & 0.501 & 0.345 & 0.472 & 0.787 \\
\hline 175 & 2.54 & 3.45 & 2 & 2.75 & 3.45 & 2.78 & 3 & 1.86 & 0.372 & 0.485 & 0.379 & 0.465 & 0.764 \\
\hline
\end{tabular}

Note: The units of the independent parameters are given in Table 7.

\subsubsection{SVR model verification}

In this process, the precision of SVR models are assessed by adopting two-stage test criterion. Firstly, 25 samples in the learning space are used to test the models according to the errors of the relative mean square root, $R M S E$ and $R^{2}$. In the II-step, the same test criterions are employed to evaluate the precision and generalisation ability of the SVR models by choosing the other 25 samples to be the prediction sets. The inspection results of SVR models are shown in Table 9. It can be given that though the precision of SVR models in the I test step is mostly higher than that in the second step, $R^{2}$ is more than $85 \%$ and RMSE is less than 0.05 respectively in the latter test step. The comparison between 
the true values of two output variables, $\rho_{1}$ and $E_{1}$ and their corresponding predictions are given in Figure 7.

Table $9 \quad \mathrm{I}$ and $\mathrm{H}$ test results of SVR models

\begin{tabular}{|c|c|c|c|c|c|c|c|c|c|}
\hline \multirow{2}{*}{\multicolumn{2}{|c|}{$\begin{array}{l}\text { SVR model } \\
\text { parameters }\end{array}$}} & \multicolumn{8}{|c|}{ Updating parameters } \\
\hline & & $\rho_{1}$ & $E_{1}$ & $E_{2}$ & $\rho_{4}$ & $E_{4}$ & $K_{1}$ & $K_{2}$ & $K_{3}$ \\
\hline \multirow{2}{*}{$\begin{array}{l}\text { I-test } \\
\text { step }\end{array}$} & $\mathrm{R}^{2}$ & 0.9905 & 0.9758 & 0.9377 & 0.9880 & 0.9918 & 0.9848 & 0.9818 & 0.9947 \\
\hline & RMSE & 0.0016 & 0.0076 & 0.0139 & 0.0026 & 0.0021 & 0.0033 & 0.0046 & 0.0001 \\
\hline \multirow{2}{*}{$\begin{array}{l}\text { H-test } \\
\text { step }\end{array}$} & $\mathrm{R}^{2}$ & 0.9947 & 0.9033 & 0.8963 & 0.9189 & 0.9202 & 0.9831 & 0.9477 & 0.9721 \\
\hline & RMSE & 0.0011 & 0.0243 & 0.0213 & 0.0173 & 0.0365 & 0.0041 & 0.0185 & 0.0056 \\
\hline
\end{tabular}

Figure 7 Precision verification of SVR model (in the H-test step), (a) comparison of true value $\rho_{1}$ and corresponding prediction (b) comparison of true value $E_{1}$ and corresponding prediction (see online version for colours)

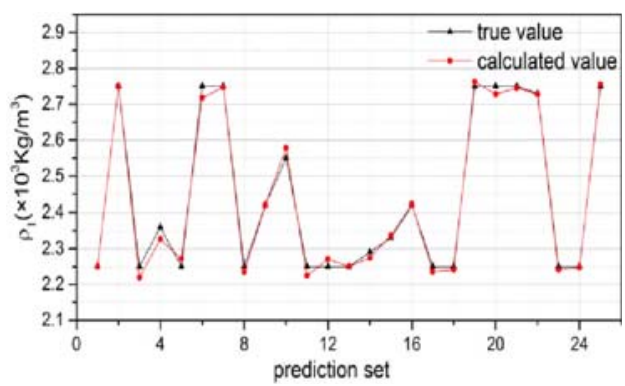

(a)

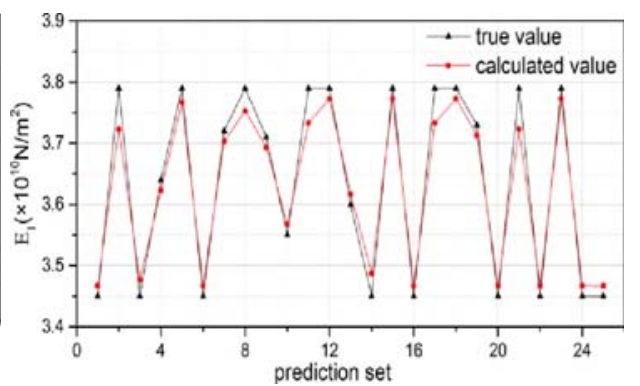

(b)

\subsubsection{Optimal design parameters}

The output variables of SVR function are considered as the updated structural design parameters and then they can be obtained by using the structural normalised measured response as the input of trained SVR function. The results of the updated parameters based on SVR and response surface model (Vapnik, 1998) are given in Table 10. Then the frequencies of the structure can be provided by using multi-scale FEM. It is indicated in Table 11 that there is less difference $(\leq 2.04 \%)$ between the true structural frequencies and these which are obtained by multi-scale FEM after the updating based on SVR method than those based on response surface model in the second stage.

Table 10 Updated structural parameters of SVR method

\begin{tabular}{lcccccccc}
\hline Updating parameters & $\rho_{1}$ & $E_{1}$ & $E_{2}$ & $\rho_{4}$ & $E_{4}$ & $K_{1}$ & $K_{2}$ & $K_{3}$ \\
\hline Initial & 2.50 & 3.45 & 2.10 & 2.50 & 3.45 & 1.00 & 1.00 & 1.00 \\
Updated based on SVR & 2.43 & 3.64 & 2.16 & 2.52 & 3.62 & 2.28 & 2.53 & 2.28 \\
Updated based on response surface & 2.46 & 3.70 & 2.28 & 2.55 & 3.70 & 2.29 & 2.83 & 2.10 \\
method & & & & & & & & \\
Updating rate based on SVR (\%) & -2.80 & 5.51 & 2.86 & 0.80 & 4.93 & 128.0 & 153.0 & 128.0 \\
$\begin{array}{l}\text { Updating rate based on response } \\
\text { surface method (\%) }\end{array}$ & -1.60 & 7.25 & 8.57 & 2.0 & 7.25 & 129.0 & 183.0 & 110.0 \\
\hline
\end{tabular}

Note: The units of the structural parameters can be found in Table 7. 
Table 11 Comparison of structural measured frequencies and the corresponding values after the updating based on SVR method and response surface method

\begin{tabular}{lccccc}
\hline Frequency (Hz) & $S 1$ & $S 2$ & $H 1$ & $H 2$ & $Z 1$ \\
\hline Measured (Liu, 2015) & 0.380 & 0.499 & 0.388 & 0.500 & 0.882 \\
Simulated before updating (1) & 0.374 & 0.489 & 0.327 & 0.447 & 0.693 \\
Simulated after updating based on SVR (2) & 0.380 & 0.500 & 0.388 & 0.491 & 0.864 \\
Simulated after updating based on response surface & 0.380 & 0.502 & 0.388 & 0.494 & 0.856 \\
method(3) & & & & & \\
Relative error before updating(1) (\%) & 1.58 & 2.00 & 15.72 & 10.60 & 21.43 \\
Relative error after updating based on SVR(2) (\%) & 0.00 & 0.20 & 0.00 & 1.80 & 2.04 \\
$\begin{array}{l}\text { Relative error after updating based on response } \\
\text { surface method(3) (\%) }\end{array}$ & 0 & 0.60 & 0.00 & 1.20 & 2.95 \\
\hline
\end{tabular}

Notes: The frequency values after the updating in the first step are used to be the frequencies before updating in the second updating step.

\section{Multi-scale FEM validation of cable-stayed bridge}

The multi-scale FEM validation of Guanhe Bridge is proceeded by employing the true frequencies obtained by SHM system of Guanhe Bridge so as to taken the uncertainty of structural parameters into consideration. The earlier obtained SVR function is taken as the surrogate model in order to discuss the inverse propagation rules. In the validation process, some validation criterions are adopted to analysis the correlation of the measured values and the corresponding computation results.

\subsection{SHM system of Guanhe Bridge}

According to SHM of Guanhe Bridge (Fan, 2014), the frequencies of twelve time fragments of the chosen four days for every month in the 2014 year are settled to obtain the 576 model frequency sample sets. These sample data are given in Table 12.

Table 12 Frequency sample data of SHM system of Guanhe Bridge

\begin{tabular}{lccccccc}
\hline \multirow{2}{*}{ Number } & \multicolumn{7}{c}{ Structural frequencies $(\mathrm{Hz})$} \\
\cline { 2 - 8 } & $S 1$ & $S 2$ & $S 3$ & $S 5$ & $H 1$ & $H 2$ & $Z 1$ \\
\hline 1 & 0.380 & 0.505 & 0.773 & 0.965 & 0.386 & 0.506 & 0.882 \\
2 & 0.380 & 0.507 & 0.778 & 0.963 & 0.387 & 0.507 & 0.892 \\
3 & 0.379 & 0.508 & 0.778 & 0.965 & 0.382 & 0.509 & 0.900 \\
$\vdots$ & $\vdots$ & $\vdots$ & $\vdots$ & $\vdots$ & $\vdots$ & $\vdots$ & \\
574 & 0.376 & 0.506 & 0.771 & 0.955 & 0.370 & 0.509 & 0.881 \\
575 & 0.378 & 0.505 & 0.773 & 0.957 & 0.376 & 0.505 & 0.881 \\
576 & 0.379 & 0.505 & 0.762 & 0.965 & 0.399 & 0.504 & 0.876 \\
\hline
\end{tabular}

Notes: The first four frequencies are the first, second, third and fifth vertical ones respectively. The other frequencies can be found in Table 8 . 


\subsection{Computation/experiment correlation analysis}

The optimised structural response can be gotten by using the updated structural parameters as the input of FEM simulation. Then the computation/experiment correlation between these response and their corresponding measured values can be obtained.

1 Relative error criterion (Zong and Ren, 2012):

$$
J(p)=\left(y_{f}-y_{\text {test }}\right) / y_{\text {test }} \times 100 \%
$$

where $y_{f}$ and $y_{\text {test }}$ are the computation result after the updating of FEM and the structural test response respectively. The results of computation/experiment correlation analysis for Guanhe Bridge is shown in Table 12. It indicates that the maximum errors between the calculated response and the measured values or the means of the measured ones are very small, just $2.48 \%$ and $3.74 \%$ respectively.

2 Correlation analysis based on modal assurance criterion (MAC)

MAC is always adopted to identify the damages and locations for structures. At present, it is common to evaluate the correlation of two mode shapes. It is defined by Oberkampf and Roy (2010)

$$
\operatorname{MAC}\left(\varphi_{f}, \varphi_{t}\right)=\frac{\left|\varphi_{f}^{T} \varphi_{t}\right|^{2}}{\left(\varphi_{f}^{T} \varphi_{f}\right)\left(\varphi_{i}^{T} \varphi_{t}\right)}
$$

where $\varphi_{f}$ and $\varphi_{i}$ represents the mode shape vectors obtained by the updated FEM and the measurement respectively. MAC, a non-dimensional parameter, is located in the interval $(0,1)$. If MAC is more close to 1 , the correlation is higher. Otherwise, the correlation is low if it is proximate to 0 .

The correlation analysis results are given in Table 14. MACs are larger than $90 \%$ and these indicate that the mode shapes obtained by the two-phase updated multi-scale FEM of Guanhe Bridge and the corresponding measured ones agree well. On the other hand, the correlation between the computed and test mode shapes is high and the updated multi-scale FEM can be adopted to reflect the structural dynamic properties.

Table 13 Computation/experiment correlation analysis based on relative error criterion

\begin{tabular}{lccccc}
\hline Frequency $(\mathrm{Hz})$ & $\begin{array}{c}\text { Measured } \\
\text { value (1) }\end{array}$ & $\begin{array}{c}\text { Measured mean } \\
\text { value (2) }\end{array}$ & $\begin{array}{c}\text { After updating based on } \\
\text { SVR (3) }\end{array}$ & $J_{1}(p)$ & $J_{2}(p)$ \\
\hline S1 & 0.380 & 0.378 & 0.380 & 0 & 0.53 \\
S2 & 0.499 & 0.504 & 0.5000 & 0.20 & -0.79 \\
S3 & 0.766 & 0.776 & 0.747 & -2.48 & -3.74 \\
S4 & 0.882 & - & 0.864 & -2.04 & - \\
S5 & 0.948 & 0.955 & 0.937 & -1.16 & -1.88 \\
H1 & 0.388 & 0.383 & 0.388 & 0 & 1.31 \\
H2 & 0.500 & 0.501 & 0.491 & -1.80 & -2.00 \\
N1 & 0.628 & - & 0.615 & -2.07 & - \\
Z1 & 0.882 & 0.876 & 0.864 & -2.04 & -1.37 \\
\hline
\end{tabular}

Notes: $J_{1}(p)$ and $J_{2}(p)$ are calculated by $J_{1}(p)=($ (3)-(1) $) /(1) \times 100 \%$ and

$J_{2}(p)=($ (3)-(2) $) /(2) \times 100 \%$ respectively. 
Table 14 MAC of mode shapes

\begin{tabular}{|c|c|c|c|c|c|}
\hline \multirow{2}{*}{ MAC (\%) } & \multicolumn{2}{|c|}{ Transverse } & \multicolumn{2}{|c|}{ Vertical } & \multirow{2}{*}{$\begin{array}{c}\text { Longitudinal } \\
\text { First order }\end{array}$} \\
\hline & First order & Second order & First order & Second order & \\
\hline SVR & 98.9 & 94.1 & 98.5 & 90.7 & 98.4 \\
\hline
\end{tabular}

\subsection{Uncertainty quantification and inverse propagation analysis (Zong and Ren, 2012)}

In this process, the main work is to discuss the inverse propagation rules of model uncertainty. In other words, the statistical rules of the uncertainty of model parameters are given by employing the uncertainty of structural characteristic response. It is common to adopt the normal distribution iterative method to accomplish this work. It adopts the direct function and provides the equation $p=f^{-1}(y)$ with the inverse of coefficient matrix. However, it is a complicated process to solve the inverse matrix if the higher order of the fitted response surface function, the more model parameters and more structural responses are taken into consideration. In order to avoid this problem, in this paper, the inverse propagation model $(p=g(y))$ based on SVR method is adopted to obtain the structural parameters according to the measured structural responses of SHM system. The measured first two vertical and transverse frequencies and the first order longitudinal frequency are adopted to be the input of the verified SVR model. Then the corresponding structural parameters can be obtained. It is assumed that all these parameters obey the normal distribution in this step. Their properties are shown in Table 15.

Table 15 Statistical values of model parameters based on SVR method

\begin{tabular}{lcccccccc}
\hline Parameters & $\rho_{1}$ & $E_{1}$ & $E_{2}$ & $\rho_{4}$ & $E_{4}$ & $K_{1}$ & $K_{2}$ & $K_{3}$ \\
\hline Mean & 2.44 & 3.69 & 2.17 & 2.57 & 3.66 & 1.98 & 2.42 & 2.24 \\
Standard deviation & 0.067 & 0.042 & 0.034 & 0.041 & 0.038 & 0.205 & 0.225 & 0.156 \\
Variation coefficient & 0.03 & 0.01 & 0.02 & 0.02 & 0.01 & 0.11 & 0.09 & 0.07 \\
\hline
\end{tabular}

Table 16 Statistical property of uncertain parameters for Guanhe Bridge

\begin{tabular}{lccccc}
\hline Parameters & Range & Mean $\mu$ & $\begin{array}{c}\text { Standard } \\
\text { deviation } \sigma\end{array}$ & $\begin{array}{c}\text { Variation } \\
\text { coefficient (\%) }\end{array}$ & $\begin{array}{c}\text { Distributed } \\
\text { type }\end{array}$ \\
\hline p1 & $2.25 \sim 2.75$ & 2.500 & 0.083 & 3.33 & Normal \\
$\mathrm{E} 1$ & $3.45 \sim 3.795$ & 3.623 & 0.058 & 1.59 & Normal \\
$\mathrm{E} 2$ & $1.995 \sim 2.31$ & 2.153 & 0.053 & 2.44 & Normal \\
$\rho 4$ & $2.25 \sim 2.75$ & 2.500 & 0.083 & 3.33 & Normal \\
$\mathrm{E} 4$ & $3.45 \sim 3.795$ & 3.623 & 0.058 & 1.59 & Normal \\
$\mathrm{K} 1$ & $1 \sim 3$ & 2.000 & 0.333 & 16.67 & Normal \\
$\mathrm{K} 2$ & $1 \sim 3$ & 2.000 & 0.333 & 16.67 & Normal \\
$\mathrm{K} 3$ & $1 \sim 3$ & 2.000 & 0.333 & 16.67 & Normal \\
\hline
\end{tabular}

Note: The unit of these parameters can be gotten in Table 6 . 
Table 17 Coupling index of structural parameters

\begin{tabular}{lcccccccc}
\hline Parameters & $\rho_{1}$ & $E_{1}$ & $E_{2}$ & $\rho_{4}$ & $E_{4}$ & $K_{1}$ & $K_{2}$ & $K_{3}$ \\
\hline With mean value error & 0.679 & 0.491 & 0.750 & 0.503 & 0.640 & 0.724 & 0.437 & 0.526 \\
Without mean value error & 0.897 & 0.845 & 0.789 & 0.670 & 0.798 & 0.770 & 0.813 & 0.650 \\
\hline
\end{tabular}

Table 18 Comparison of parameters after consideration of the uncertainty

\begin{tabular}{lcccccccc}
\hline Parameters & $\rho_{1}$ & $E_{1}$ & $E_{2}$ & $\rho_{4}$ & $E_{4}$ & $K_{1}$ & $K_{2}$ & $K_{3}$ \\
\hline Initial1) & 2.50 & 3.45 & 2.10 & 2.50 & 3.45 & 1.00 & 1.00 & 1.00 \\
Updated(2) & 2.43 & 3.64 & 2.16 & 2.52 & 3.62 & 2.28 & 2.53 & 2.28 \\
Statistical mean value(3) & 2.44 & 3.69 & 2.17 & 2.57 & 3.66 & 1.98 & 2.42 & 2.24 \\
Deviation a (\%) & 2.40 & 6.96 & 3.33 & 2.8 & 6.09 & 98 & 142 & 124 \\
Deviation b (\%) & 0.41 & 1.37 & 0.46 & 1.98 & 1.10 & 13.16 & 4.35 & 1.75 \\
\hline
\end{tabular}

Notes: The deviation a and $\mathrm{b}$ can be calculated by $a=($ (3)-(1) $) /(1) * 100 \%$ and $b=(3-$ (2) $) /(2) * 100 \%$ respectively.

Figure 8 Comparison of the initial and computed PDF of structural parameters, (a) parameter $\rho_{1}$ (b) parameter $E_{1}(\mathrm{c})$ parameter $E_{2}(\mathrm{~d})$ parameter $\rho_{4}(\mathrm{e})$ parameter $E_{4}(\mathrm{f})$ parameter $K_{1}$ (g) parameter $K_{2}(\mathrm{~h})$ parameter $K_{3}$ (see online version for colours)

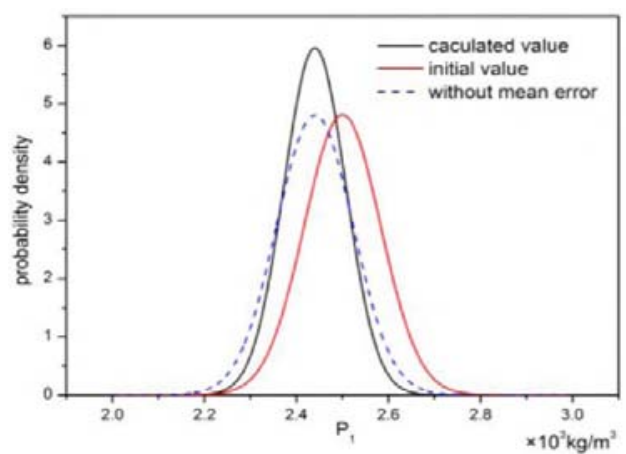

(a)

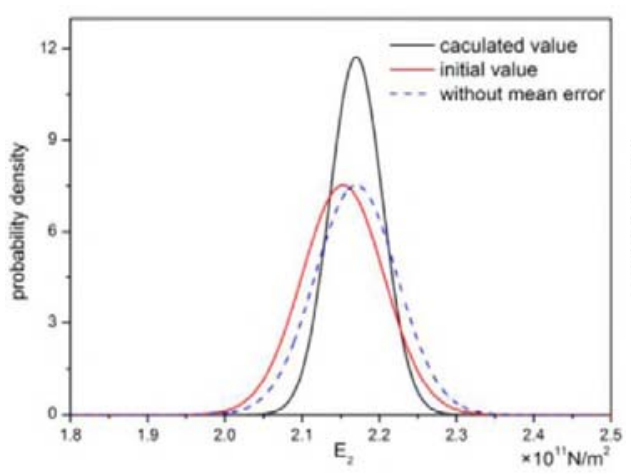

(c)

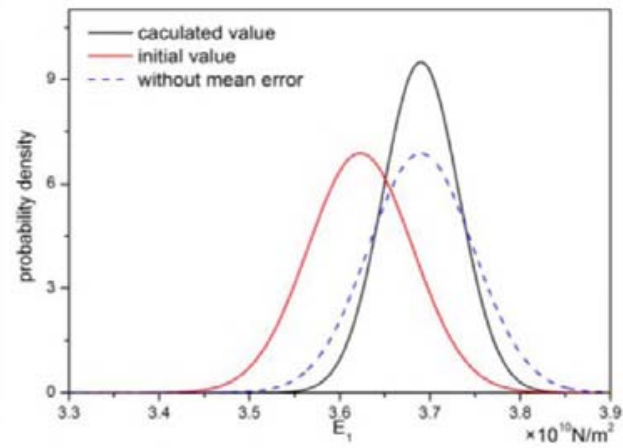

(b)

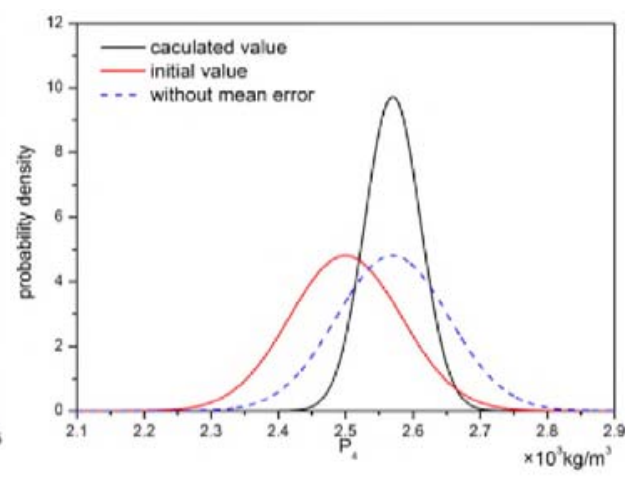

(d) 
Figure 8 Comparison of the initial and computed PDF of structural parameters, (a) parameter $\rho_{1}$ (b) parameter $E_{1}(\mathrm{c})$ parameter $E_{2}(\mathrm{~d})$ parameter $\rho_{4}(\mathrm{e})$ parameter $E_{4}(\mathrm{f})$ parameter $K_{1}$ (g) parameter $K_{2}(\mathrm{~h})$ parameter $K_{3}$ (continued) (see online version for colours)

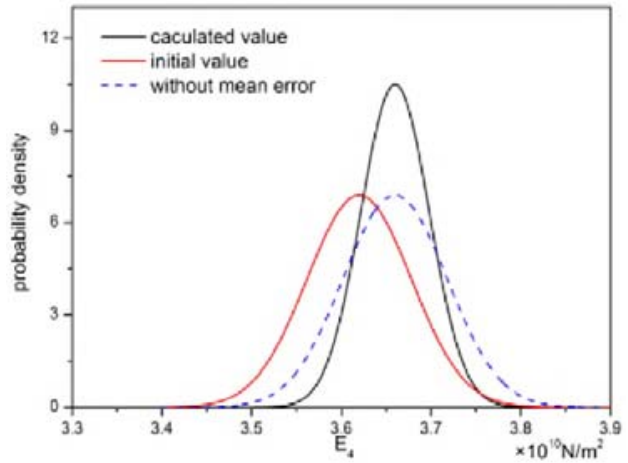

(e)

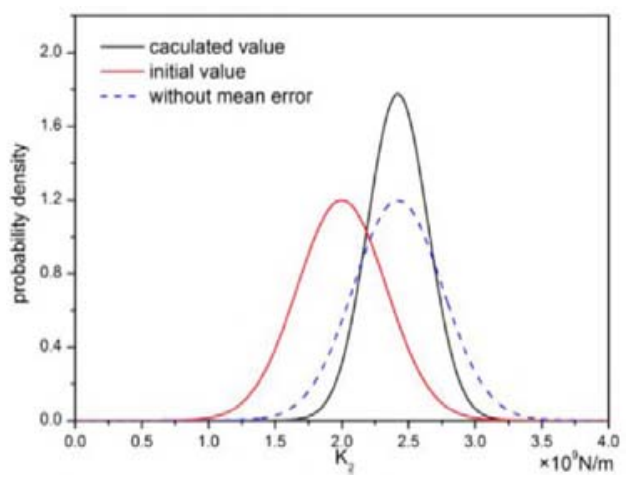

(g)

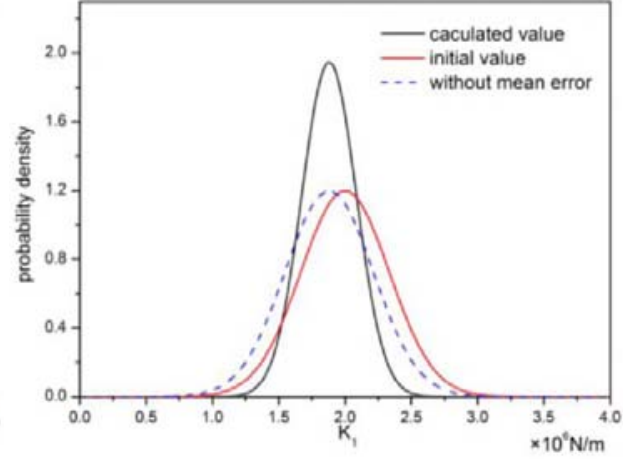

(f)

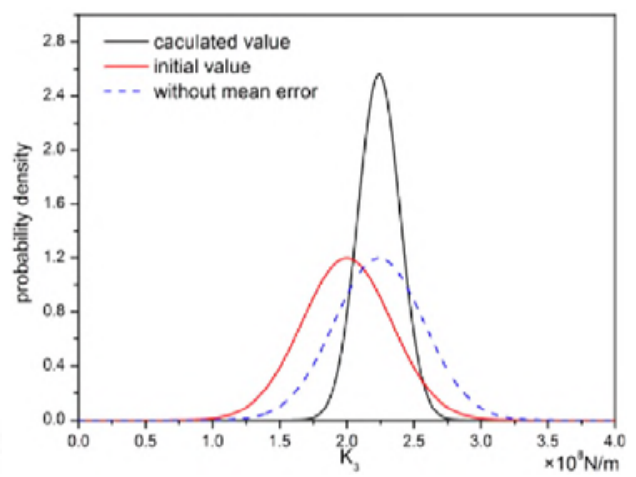

(h)

For Guanhe Bridge, the initial statistical condition of the structural parameters can be defined by combing the initial range of the parameters in Table 6 and $3 \sigma$ principle and then it is considered as their initial distribution. The statistical results are given in Table 16. Their corresponding probability density function (PDF) of computed/initial values for these parameters can be computed to obtain the coupling area of the PDF of the structural parameters. The coupling analysis consequences are provided in Table 17 and Figure 8. It is shown in Table 16 that there are two factors, mean value error and standard deviation error, for the low coupling results between the parameters computed by SVR model and the initial structural parameters. The former error results from the model updating inevitably and it can be removed firstly in order to analyse the coupling again. The new coupling indexes in Table 17 are more than $65 \%$. Similarly, it indicates in Figure 8 that it is small for the standard deviation of the structural parameters which is computed by adopting SVR inverse propagation rule. This condition has a big relationship with the low discreteness of SHM data. So they agree well. The statistic results of structural parameters are shown in Table 18 after taking the uncertainty of the structural response into consideration. There is a low deviation $(<5 \%)$ mostly between the statistical mean values and the updated optimal parameters. So the more accurate range of 
the structural parameters can be obtained if the uncertainty of structural response is taken into consideration.

Some of the normal test results of the eight structural parameters are given in Figure 9. There is a certain discreteness because the measured frequencies are adopted to calculate the structural parameters. However, the samples of structural parameters are close to the test line mostly according to the left probability distribution test figure. So the structural parameters based on SVR method obey the assumed normal distribution approximately. Finally, the validated multi-scale FEM of Guanhe Bridge can be adopted to do the further work of structural damage prognosis and safety prognosis.

Figure 9 Normal distribution verification based on SVR simulated data, (a) normal probability distribution verification of simulation data of $\rho_{1}$ (b) comparison of the empirical and theoretical distribution function of $\rho_{1}$ (c) normal probability distribution verification of simulation data of $E_{1}(\mathrm{~d})$ comparison of the empirical and theoretical distribution function of $E_{1}$ (e) normal probability distribution verification of simulation data of $\rho_{4}$ (f) comparison of the empirical and theoretical distribution function of $\rho_{4}(\mathrm{~g})$ normal probability distribution verification of simulation data of $K_{1}(\mathrm{~h})$ comparison of the empirical and theoretical distribution function of $K_{1}$ (i) normal probability distribution verification of simulation data of $K_{3}(\mathrm{j})$ comparison of the empirical and theoretical distribution function of $K_{3}$ (see online version for colours)

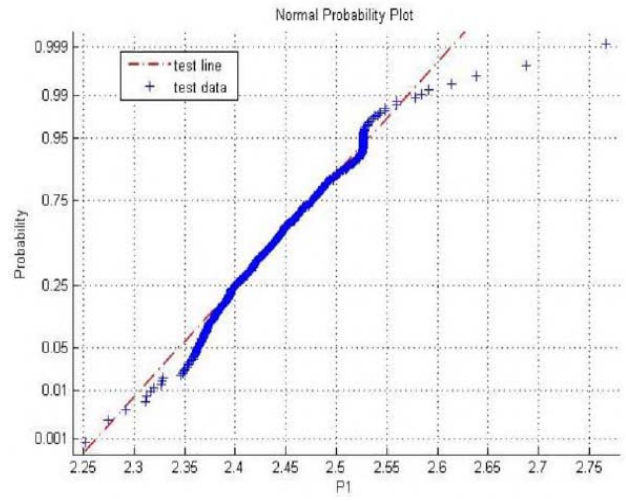

(a)

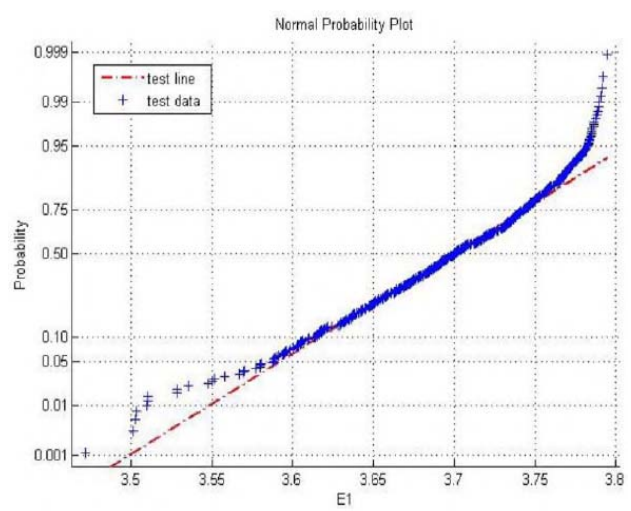

(c)

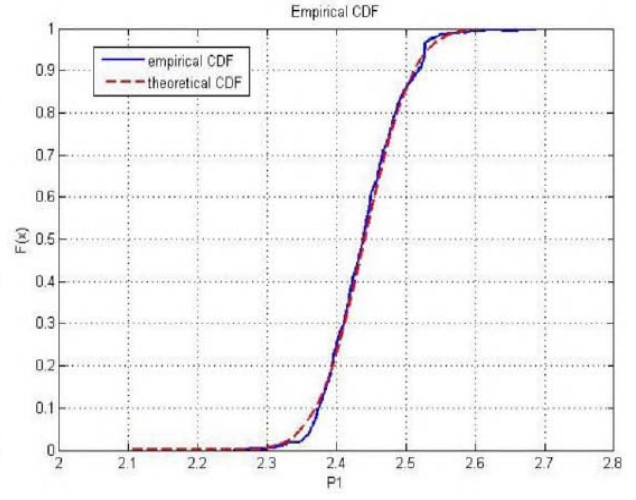

(b)

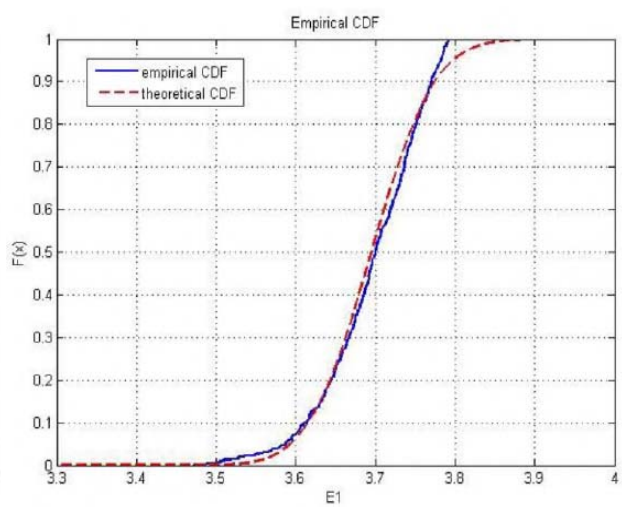

(d) 
Figure 9 Normal distribution verification based on SVR simulated data, (a) normal probability distribution verification of simulation data of $\rho_{1}$ (b) comparison of the empirical and theoretical distribution function of $\rho_{1}$ (c) normal probability distribution verification of simulation data of $E_{1}(\mathrm{~d})$ comparison of the empirical and theoretical distribution function of $E_{1}$ (e) normal probability distribution verification of simulation data of $\rho_{4}$ (f) comparison of the empirical and theoretical distribution function of $\rho_{4}(\mathrm{~g})$ normal probability distribution verification of simulation data of $K_{1}(\mathrm{~h})$ comparison of the empirical and theoretical distribution function of $K_{1}$ (i) normal probability distribution verification of simulation data of $K_{3}(\mathrm{j})$ comparison of the empirical and theoretical distribution function of $K_{3}$ (continued) (see online version for colours)

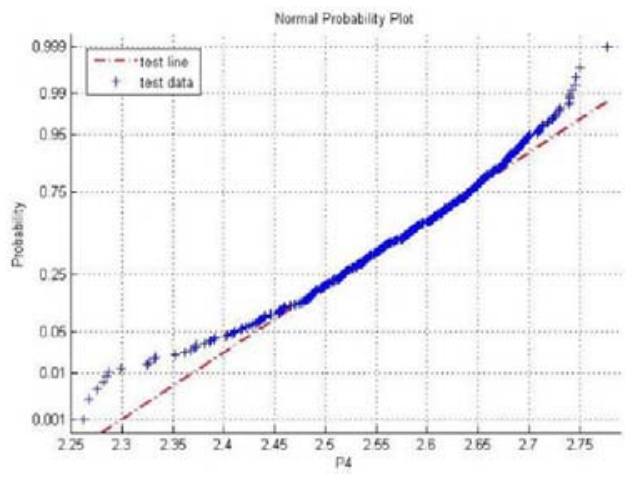

(e)

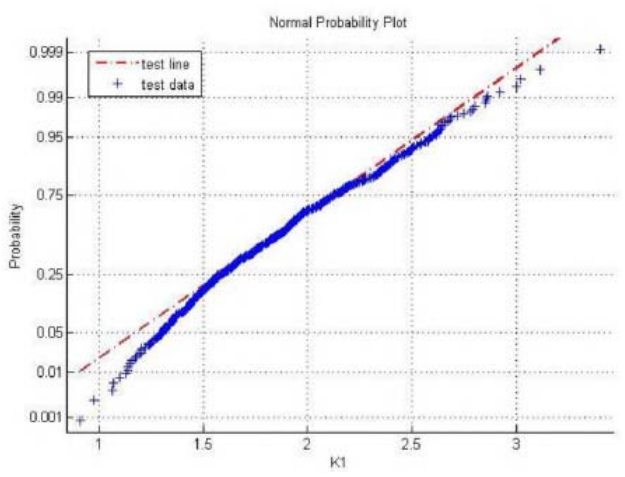

(g)

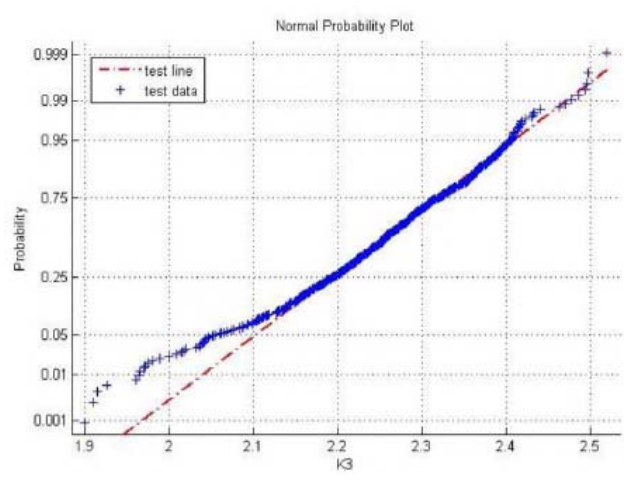

(i)

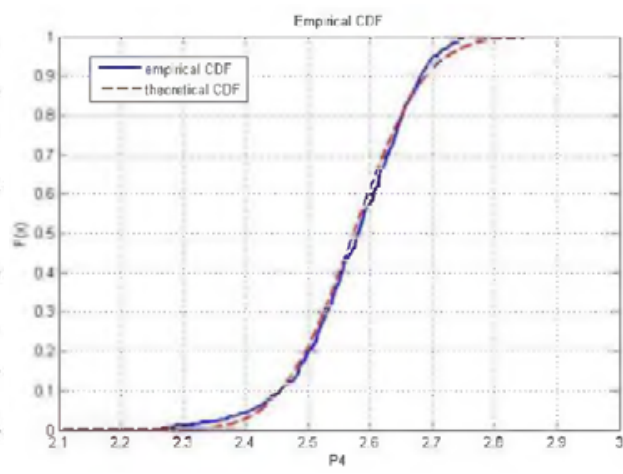

(f)

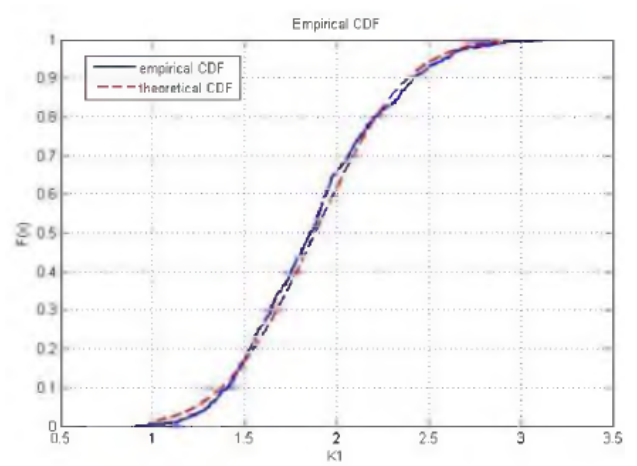

(h)

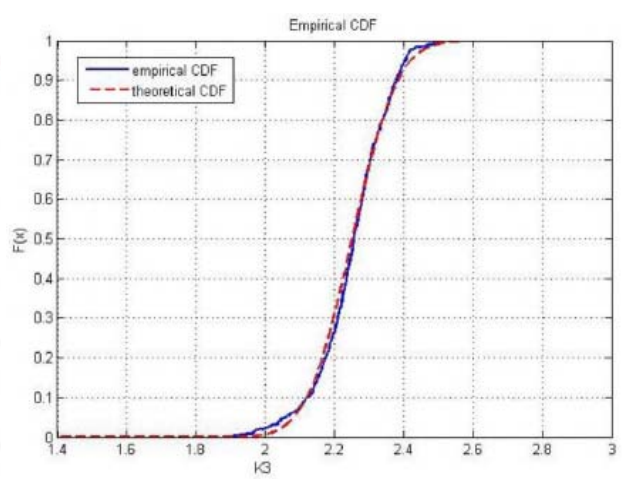

(j) 


\section{Conclusions}

In this paper, a new method based on two-phase response surface method and SVR method was proposed to validate the multi-scale FEM. Its feasibility is verified by its application in the multi-scale FEM validation of a composite cable-stayed bridge, Guanhe Bridge. The conclusions are as follows:

1 Multi-scale FEM of Guanhe Bridge is established by adopting structural multi-scale simulation method based on Arlequin.

2 Two-phase structural multi-scale FEM updating method is proposed according to the multi-scale FEM of Guanhe Bridge. The updating results indicate that there is less error $(<2.1 \%)$ between the frequencies based on multi-scale model based on SVR method than those based on response surface model in the second stage and the corresponding measured ones. So the presented method can be adopted to update the multi-scale FEM of composite cable-stayed bridge.

3 The statistical results of structural parameters are gotten by using the inverse computation of the uncertainty of structural response. It is shown that the coupling indexes of all the structural parameters are larger than $65 \%$. Most of the deviations between the statistical mean values and the corresponding updated optimal parameters is small $(<5 \%)$. So the accurate structural parameters can be obtained after the consideration of the uncertainty of structural response.

4 The distribution of structural parameters based on SVR function is verified. The statistical results show that the parameters obey the supposed normal distribution on the whole. So the validated multi-scale FEM can be adopted to precede the structural damage prognosis and safety prognosis.

However, the multi-input and single-output SVR method was adopted to update and validate the multi-scale FEM in this paper. The correlation of the output parameters was not taken into consideration in this process. So the research about these two problems is still needed in the further work.

\section{Acknowledgements}

The authors gratefully acknowledge the financial support provided by the Natural Science Foundation of China (Grant No. 51678141) and the Priority Academic Program Development of Jiangsu Higher Education Institutions (PAPD). The viewpoints of this paper represent only the author's opinion, do not represent the views of the fund committee. 


\section{References}

Adhikari, S. and Sarkar, A. (2009) 'Uncertainty in structural dynamics: experimental validation of a Wishart random matrix model', Journal of Sound and Vibration, Vol. 323, Nos. 3-5, pp.802-825.

Atamturktur, H.S., Moaveni, B., Papadimitriou, C. et al. (2015) 'Model validation and uncertainty quantification', Proceedings of the 33rd IMAC, A Conference and Exposition on Structural Dynamics, Springer, New York, Vol. 3.

Batou, A., Soize, C. and Audebert, S. (2015) 'Model identification in computational stochastic dynamics using experimental modal data', Mechanical System \& Signal Process, Vols. 50-51, pp.307-322.

Ben, D.H. (1998) 'Multiscale mechanical problems: the Arlequin method', Comptes Rendus de l'Academie des Sciences Series IIB Mecanique Physique Astronomie, Vol. 326, No. 12, pp.899-904.

Catbas, N., Gokce, H.B. and Frangopol, D.M. (2013) 'Predictive analysis by incorporating uncertainty through a family of models calibrated with structural health-monitoring data', Journal of Engineering Mechanics, Vol. 139, No. 6, pp.712-723.

Chan, T.H.T., Li, Z.X., Yu, Y. et al. (2009) 'Concurrent multi-scale modeling of civil infrastructures for analyses on structural deteriorating-part II: model updating and verification', Finite Elements in Analysis and Design, Vol. 45, No. 11, pp.795-805.

Chen, W., Lusine, B., Thaweepat, B. et al. (2004) 'Model validation via uncertainty propagation and data transformations', AIAA Journal, Vol. 42, No. 7, pp.1406-1415.

Ding, Y.L., Li, A.Q., Du, D.S. et al. (2010) 'Multi-scale damage analysis for a steel box girder of a long-span cable-stayed bridge', Structure and Infrastructure Engineering, Vol. 6, No. 6, pp.725-739.

Efendiev, Y. and Hou, T.Y. (2009) Multiscale Finite Element Methods: Theory and Applications, Springer London Ltd, London.

Fan, X.C. (2014) Research on Safety Alarming Method of Steel-Concrete Composite Beam Cable-Stayed Bridge Based on Structural Health Monitoring, Chinese, Southeast University.

Farrar, C.R. and Lieven, N.A.J. (2007) 'Damage prognosis: the future of structural health monitoring', Philosophical Transactions of the Royal Society A, Vol. 365, No. 1851, pp.623-632.

Greco, F., Leonetti, L., Lonetti, P. et al. (2015) 'Crack propagation analysis in composite materials by using moving mesh and multiscale techniques', Computers \& Structures, Vol. 153, pp.201-216.

Hemez, F.M. and Doebling, S.W. (2001) 'Model validation and uncertainty quantification', Proceedings of IMAC-XIX, The 19th International Modal Analysis Conference, pp.6-19.

Jaishi, B. and Ren, W.X. (2007) 'Finite element model updating based on eigenvalue and strain energy residuals using multiobjective optimization technique', Mechanical Systems and Signal Processing, Vol. 21, No. 5, pp.2295-2317.

Liu, Q.Q. (2015) Research on Multi-Scale Finite Element Model Updating and Validation Method of Cable-Stayed Bridge, Chinese, Southeast University.

Liu, W.K., Besterfield, G.H. and Belytschko, T. (1988) 'Variational approach to probabilistic finite elements', Journal of Engineering Mechanics, Vol. 114, No. 12, pp.2115-2133.

Liu, W.K., Qian, D., Gonella, S. et al. (2010) 'Multiscale methods for mechanical science of complex material: bridging from quantum to stachastic multiresolution continuum', International Journal for Numerical methods in Engineering, Vol. 83, Nos. 8-9, pp.1039-1080

Mak, K.H., Popov, A.A. and Mc, W.S. (2012) 'Experimental model validation for a nonlinear energy harvester incorporating a bump stop', Journal of Sound and Vibration, Vol. 331, No. 11, pp.2602-2623. 
Marwala, T. (2010) Finite Element Model Updating Using Computational Intelligence Techniques: Applications to Structural Dynamics, Springer Publishing Company, London.

Michaels, J.E., Jacobs, L.J. and Ruzzene, M. (2012) Multi-scale Structural Health Monitoring Approach for Damage Detection, Diagnosis and Prognosis in Aerospace Structures, Georgia Tech Research Corporation, Atlanta.

Montomoli, F., Camevale, M., D'Ammaro, A. et al. (2015) Uncertainty Quantification in Computational Fluid Dynamics and Aircraft Engines, Springer, New York.

Moradi, S., Fatahi, L. and Razi, P. (2010) 'Finite element model updating using bees algorithm', Structural and Multidisciplinary Optimization, Vol. 42, No. 2, pp.283-291.

Nishio, M., Marin, J. and Fujino, Y. (2012) 'Uncertainty quantification of the finite element model of existing bridges for dynamic analysis', Journal of Civil Structural Health Monitoring, Vol. 2, Nos. 3-4, pp.163-173.

Oberkampf, W. and Roy, C. (2010) Verification and Validation in Scientific Computing, Cambridge University Press, Cambridge.

Ou, J.P. and Li, H. (2010) 'Structural health monitoring in mainland China: review and future trends', Structural Health Monitoring, Vol. 9, No. 3, pp.219-231.

Paez, T.L. and Red-Horse, J. (2008) 'Structural dynamics challenge problem: summary', Computer Methods in Applied Mechanics and Engineering, Vol. 197, Nos. 29-32, pp.2660-2665.

Perera, R., Roberto, M. and Ruiz, A. (2013) 'Static-dynamic multi-scale structural damage identification in a multi-objective framework', Journal of Sound and Vibration, Vol. 332, No. 6, pp.1484-1500.

Qiao, H., Yang, Q.D., Chen, W.Q. et al. (2011) 'Implementation of the Arlequin method into ABAQUS: Basie formulations and applications', Advances in Engineer Software, Vol. 42, No. 4, pp.197-207.

Red-Horse, J. and Paez, T.L. (2008) 'Sandia National Laboratories validation workshop: structural dynamics application', Computer Methods in Applied Mechanics and Engineering, Vol. 197, Nos. 29-32, pp.2578-2584.

Ren, W.X. and Chen, H.B. (2010) 'Finite element model updating in structural dynamics by using the response surface method', Engineering Structures, Vol. 32, No. 8, pp.2455-2465.

Renno, J.M. and MacE, B.R. (2012) 'Vibration modelling of helical springs with non-uniform ends', Journal of Sound and Vibration, Vol. 331, No. 12, pp.2809-2823.

Smith, R.S. (1995) 'Model validation for robust control: an experimental process control application', Automatica, Vol. 31, No. 11, pp.1637-1647.

Smith, R.S. and Doyle, J.C. (1992) 'Model validation: a connection between robust control and identification', IEEE Transactions on Automatic Control, Vol. 37, No. 7, pp.942-952.

Starge, D. (2010) Multi-scale Structural Mechanics and Prognosis, Program Manager AFOSR/ RTA, Air Force Research Laboratory, Virginia.

Takizawa, K. and Tazduyar, T.E. (2011) 'Multiscale space-time fluid-structure interaction techniques', Computational Mechanics, Vol. 48, No. 3, pp.247-267.

Thompson, D.J., Vincent, N., Gautier, P.E. (1999) 'Validation of a model for railway rolling noise using field measurements with sinusoidally profiled wheels', Journal of Sound and Vibration, Vol. 223, No. 4, pp.587-609.

Vapnik, V.N. (1998) Statistical Learning Theory, A Wiley Inter-science Publication, John Wiley \& Sons, New York.

Wei, S. and Dyke, S. (2014) 'Real-time dynamic model updating of a hysteretic structural system', Journal of Structural Engineering, Vol. 140, No. 3, p.4013082-1-14.

Xiao, X., Xu, Y.L. and Zhu, Q. (2015) 'Multiscale modeling and model updating of a cable-stayed bridge. II: model updating using modal frequencies and influence lines', Journal of Bridge Engineering, Vol. 20, No. 10, p.4014113-1-04014113-12. 
Yang, Y. (2012) Multi-Scale Modelling of Long-Span Bridges for Health Assessment in Structural Health Monitoring, The Hong Kong Polytechnic University, Hong Kong.

Zhong, R.M., Fan, X.C., Huang, X.Y. et al. (2013) 'Multi-scale finite element model updating of composite cable-stayed bridge based on the two-phase response surface methods', Journal of Southeast University, Chinese, Vol. 43, No. 5, pp.993-999.

Zhong, R.M., Zong, Z.H., Liu, Q.Q. et al. (2015) 'A multiscale finite element model validation of composite cable-stayed bridge based on structural health monitoring system', Shock and Vibration, Vol. 2015, Article ID 817281, pp.1-12.

Zhong, R.M., Zong, Z.H., Niu, J. et al. (2016) 'A multiscale finite element model validation method of composite cable-stayed bridge based on probability box theory', Journal of Sound and Vibration, Vol. 370, pp.111-131.

Zong, Z.H. and Ren, W.X. (2012) Finite Element Model Updating \& Validation of Bridge Structures, People Transportation Press, Beijing.

Zong, Z.H., Zhong, R.M., Zheng, P.J. et al. (2014) 'Damage \& safety prognosis of bridge structures based on the structural health monitoring: progress and challenges', China Journal of Highway and Transport, Chinese, Vol. 27, No. 12, pp.46-57. 